\title{
Re: Is Anastomotic Urethroplasty is Really Superior Than BMG Augmented Dorsal Onlay Urethroplasty in Terms of Outcomes and Patient Satisfaction: Our 4-Year Experience
}

\author{
Anıl Kumar Choudhary1, Nawal K. Jha2 \\ 1 Ram Manohar Lohia Hospital, New Delhi, India \\ 2Rajendra Institute of Medical Sciences, Ranchi, India
}

Can Urol Assoc J 2015;9:E22-26. doi: 10.5489/cuaj.2291.

\section{EDITORIAL COMMENT}

The ideal bulbar urethral stricture management technique has not yet been found. Various treatment methods can be used successfully in bulbar urethral stricture, and surgeons must be comfortable with different procedures, as anatomical pathology can vary intraoperatively. The art of managing urethral stricture disease requires that reconstructive urologists be facile with multiple techniques that can be tailored to each patient. In this article, the study kindly presented the authors' experience in carrying out two different variations on repair of bulbar urethral stricture. The authors compared the effectiveness of buccal mucosa graft dorsal onlay urethroplasty (BMG) with excision and primary anastomosis. Over 75\% of these strictures were of traumatic or iatrogenic etiologies, and none of the patients were redo urethroplasties and none had hypospadias. The authors demonstrated that these techniques can achieve similar functional outcomes, despite the BMG group had longer strictures $(5.9 \mathrm{~cm}$ vs. $<2 \mathrm{~cm}$ ). Just as importantly, the authors demonstrated a reduced complication rate with BMG. Due to advances in surgical techniques, the success of urethral reconstruction is measured in quality of life, which includes return to normal urinary and sexual function. While the ultimate success of an operation is highly dependent on surgical skill, the authors concluded that BMG may help improve patient outcomes for any length of bulbar stricture needing urethroplasty.

Çetin Demirdağ MD

\section{Re: Prostatectomy for Localized Prostate Cancer to Prepare for Renal Transplantation in End-Stage Renal Disease Patients}

\author{
Xavier Tillou, Charles Chahwan, Sophie Le Gal, Henri Bensadoun, Arnaud Doerfler
}

CHU Cote de Nacre, Department of Urology and Transplantation, Caen, France

Ann Transplant 2014;19:569-575.

\section{EDITORIAL COMMENT}

The presence of prostate cancer (PC) is a real challenge in patients listed for kidney transplantation, because there are no specific recommendations for the treatment of these patients. The role of the urologist is to provide a cure for the patient by allowing inclusion to the waiting list after ascertaining the absence of disease recurrence. In theory, this period is 5 years without biochemical recurrence, a period based on data from a study evaluating the risk of recurrence after transplantation in around 18\% of patients who underwent prostatectomy before organ transplantation. After stratifying the patients according to stage of the disease, a 2-year safety period had been suggested for low-risk patients. In this retrospective study, the authors evaluated 19 patients diagnosed with low and intermediate risk of PC according to the d'Amico classification before renal transplantation over a 10-year period. All had radical prostatectomy prior to transplantation, however, only 14 were transplanted after a mean of 32.8 months from prostatectomy. Seven recipients (50\%) were transplanted less than 24 months after prostatectomy. No recurrence of PC was observed after renal transplantation, with a mean follow-up of 38 months. Despite increased difficulties in dissection during renal transplantation, a history of radical prostatectomy was not found to affect the implementation of a transplant. Despite being the largest cohort, still the numbers are very limited and the authors have carefully concluded that radical prostatectomy poses no serious surgical problems during transplantation and ensures the best oncologic follow-up before and after kidney transplantation. For low-risk PC, waiting time should be 1 year, 2 years for intermediate-risk PC, and 5 years for high risks of recurrence.

\section{REFERENCES}

1. Penn I. Evaluation of transplant candidates with pre-existing malignancies. Ann Transplant 1997;2:14-17. 